\title{
29. MESOZOIC FORAMINIFERA, LEG 22, SITE 217
}

\author{
Emile A. Pessagno, Jr., Geosciences Division, University of Texas at Dallas, Dallas, Texas \\ and
}

Fouad Y. Michael, Atlantic Richfield Company, Geological Science Group, Dallas, Texas

$\mathrm{TA}=$ Total abundance of all planktonic foraminifera in the sample.

RA $=$ Relative abundance of each taxon.

\begin{tabular}{|c|c|}
\hline \multicolumn{2}{|c|}{$22-217-17-1(68-70 \mathrm{~cm})$} \\
\hline RA & \\
\hline 2 & Gublerina robusta de Klasz \\
\hline 3 & Heterohelix sp. aff. H. striata (Ehrenberg) \\
\hline 4 & Planoglobulina brazoensis Martin \\
\hline 4 & Planoglobulina carseyae Plummer \\
\hline 3 & Pseudoguembelina kempensis Esker \\
\hline 1 & Pseudoguembelina sp. aff.P. cornata Seiglie \\
\hline 2 & Pseudoguembelina sp. \\
\hline 4 & Pseudotextularia deformis (Kikoine) \\
\hline 3 & Pseudotextularia elegans s.l. (Rzehak) \\
\hline 2 & Pseudotextularia sp. aff. $P$. intermedia de Klasz \\
\hline 4 & Racemiguembelina fructicosa (Egger) \\
\hline 1 & Racemiguembelina powelli Smith and Pessagno? \\
\hline 2 & Ventilabrella multicamerata de Klasz \\
\hline 1 & Globigerinelloides sp. \\
\hline 4 & Globotruncana arca (Cushman) \\
\hline 1 & Globotruncana calcarata Cushman (Reworked) \\
\hline 3 & Globotruncana conica White \\
\hline 2 & Globotruncana contusa (Cushman) \\
\hline 4 & Globotruncana elevata Brotzen \\
\hline 3 & Globotruncana gansseri Bolli \\
\hline 4 & Globotruncana nothi (Bronnimann and Brown) \\
\hline 3 & Globotruncana sp. \\
\hline 2 & Globotruncana stuartiformis Dalbiez \\
\hline 1 & Globotruncana trinidadensis Gandolfi \\
\hline 1 & Rugoglobigerina pennyi Bronnimann \\
\hline 1 & Rugoglobigerina sp. \\
\hline 2 & Abathomphalus mayaroensis (Bolli) \\
\hline 2 & Abathomphalus intermedius (Bolli) \\
\hline 3 & Bolivinoides draco (Marsson) (Rewor \\
\hline
\end{tabular}

Biostratigraphic determination: Globotruncana contusastuartiformis Assemblage Zone, Abathomphalus mayaroensis Subzone. Latest Maastrichtian, with reworking of latest Campanian.

$$
\text { 22-217-17-3 (64-66 cm) }
$$

TA: 0

Barren

22-217-18-2 (80-82 cm)

RA

1 Heterohelix globulosa (Ehrenberg)

1 Globigerinelloides subcarinatus (Bronnimann)

TA: 1
22-217-19-1 (80-82 cm)

TA: 2

\section{RA}

1 Gublerina robusta de Klasz

1 Heterohelix striata (Ehrenberg)

1 Heterohelix globulosa (Ehrenberg)

1 Pseudoguembelina kempensis Esker

2 Globotruncana arca (Cushman)

1 Globotruncana conica White

1 Globotruncana contusa (Cushman)

2 Globotruncana elevata (Brotzen)

1 Globotruncana plummerae Gandolfi ?

1 Rugoglobigerina pennyi Bronnimann

1 Rugoglobigerina rugosa (Plummer)

1 Globotruncanella sp. a Smith and Pessagno

2 Abathomphalus mayaroensis (Bolli)

1 Abathomphalus intermedius (Bolli)

Biostratigraphic determination: Globotruncana contusastuartiformis Assemblage Zone, Abathomphalus mayaroensis Subzone. Latest Maastrichtian.

\section{2-217-19-2 (80-82 cm) TA: 2}

\section{RA}

1 Heterohelix striata (Ehrenberg)

1 Planoglobulina carseyae (Plummer)

1 Pseudoguembelina kempensis Esker

1 Pseudoguembelina palpebra Bronnimann and Brown?

1 Racemiguembelina fructicosa (Egger)

1 Globotruncana arca (Cushman)

1 Globotruncana conica White

1 Rugoglobigerina pennyi Bronnimann

1 Abathomphalus mayaroensis (Bolli)

Biostratigraphic determination: Globotruncana contusastuartiformis Assemblage Zone, Abathomphalus mayaroensis Subzone. Latest Maastrichtian.

\section{2-217-19-3 (80-82 cm) TA: 4}

2 Gublerina robusta de Klasz

2 Heterohelix striata (Ehrenberg)

1 Heterohelix navarroensis Loeblich?

1 Planoglobulina brazoensis Martin

2 Planoglobulina carseyae Plummer

2 Pseudoguembelina palpebra Bronnimann and

Brown?

$1 \quad$ Pseudotextularia elegans (Rzehak)

4 Racemiguembelina fructicosa (Egger)

1 Racemiguembelina powelli Smith and Pessagno

1 Ventilabrella multicamerata de Klasz 
4 Globotruncana arca (Cushman)

4 Globotruncana conica White

1 Globotruncana contusa (Cushman)

4 Globotruncana elevata (Brotzen)

1 Globotruncana trinidadensis Gandolfi

2 Rugoglobigerina pennyi Bronnimann

1 Rugoglobigerina rugosa (Plummer)

2 Abathomphalus mayaroensis (Bolli)

1 Abathomphalus intermedius (Bolli)

Biostratigraphic determination: Same as previous sample. Latest Maastrichtian.

\section{2-217-20-3 (80-82 cm)}

TA: 4

RA

1 Gublerina robusta de Klasz

2 Heterohelix striata (Ehrenberg)

1 Planoglobulina carseyae (Plummer)

1 Pseudoguembelina kempensis Esker

1 Pseudotextularia deformis (Kikoine)

1 Pseudotextularia elegans (Rzehak)

1 Racemiguembelina fructicosa (Egger)

3 Globotruncana arca (Cushman)

2 Globotruncana conica White

3 Globotruncana elevata (Brotzen)

2 Globotruncana contusa (Cushman)

1 Rugoglobigerina pennyi Bronnimann

2 Rugoglobigerina rugosa (Plummer)

2 Abathomphalus mayaroensis (Bolli)

1 Abathomphalus intermedius (Bolli)

Biostratigraphic determination: Globotruncana contusastuartiformis Assemblage Zone, Abathomphalus mayaroensis Subzone. Latest Maastrichtian.

\section{2-217-21-3 (66-68 cm)}

TA: 4

RA

4 Gublerina robusta de Klasz

3 Heterohelix striata (Ehrenberg)

3 Planoglobulina brazoensis Martin

3 Planoglobulina carseyae (Plummer)

2 Pseudoguembelina kempensis Esker

3 Pseudotextularia elegans (Rzehak)

1 Racemiguembelina powelli Smith and Pessagno

2 Racemiguembelina $\mathrm{sp}$. ?

2 Ventilabrella multicamerata de Klasz

4 Globotruncana arca (Cushman)

2 Globotruncana nothi (Bronnimann and Brown)

3 Globotruncana elevata (Brotzen)

2 Globotruncana plummerae Gandolfi ?

2 Globotruncana trinidadensis Gandolfi

2 Globotruncana sp.?

3 Rugoglobigerina hexacamerata Bronnimann

3 Rugoglobigerina pennyi Bronnimann

2 Rugoglobigerina rugosa (Plummer)

3 Abathomphalus mayaroensis (Bolli)

Biostratigraphic determination: Globotruncana contusastuartiformis Assemblage Zone, Abathomphalus mayaroensis Subzone. Late Maastrichtian.
22-217-21-5 (70-72 cm)

TA: 5

RA

3

3

2

2

1

3

3

1

2

2

1

1

2

4

1

4

1

3

2

1

3

1

4

2

3

2

3

2

Gublerina robusta de Klasz

Heterohelix globulosa (Ehrenberg)

Heterohelix navarroensis Loeblich

Heterohelix striata (Ehrenberg)

Planoglobulina acervulinoides (Egger)

Planoglobulina brazoensis Martin

Planoglobulina carseyae (Plummer)

Pseudoguembelina excolata (Cushman)

Pseudoguembelina kempensis Esker

Pseudotextularia deformis (Kikoine)

Pseudotextularia elegans (Rzehak) (Reworked?)

Racemiguembelina intermedia (de Klasz)

Ventilabrella multicamerata de Klasz

Globigerinellaides prairehillensis Pessagno

Globotruncana aegyptiaca Nakkady?

Globotruncana arca (Cushman)

Globotruncana conica White

Globotruncana elevata (Brotzen)

Globotruncana patelliformis Gandolfi

Globotruncana plummerae Gandolfi

Globotruncana trinidadensis Gandolfi

Globotruncana stuartiformis Dalbiez

Rugoglobigerina pennyi Bronnimann

Rugoglobigerina rugosa (Plummer)

Globotruncanella monmouthensis (Olsson)

Globotruncanella sp.

Abathomphalus mayaroensis (Bolli)

Abathomphalus intermedius (Bolli)

Biostratigraphic determination: Globotruncana contusastuartiformia Assemblage Zone, Abathomphalus mayaroensis Subzone. Late Maastrichtian.

\section{2-217-22-1 (80-82 cm) TA: 5}

RA

3 Gublerina robusta de Klasz

3 Heterohelix punctulata (Cushman)

3 Heterohelix striata (Ehrenberg)

3 Planoglobulina brazoensis Martin

3 Planoglobulina carseyae (Plummer)

1 Pseudoguembelina sp.

3 Pseudotextularia deformis (Kikoine)

2 Pseudotextularia elegans (Rzehak)

2 Racemiguembelina fructicosa (Egger)

3 Globotruncana arca (Cushman)

1 Globotruncana contusa (Cushman)

2 Globotruncana elevata (Brotzen)

2 Globotruncana lapparenti Brotzen (Reworked)

2 Globotruncana linneiana (d'Orbigny) (Reworked)

1 Globotruncana stuarti (de Lapparent)

1 Globotruncana stuartiformis Dalbiez ?

4 Rugoglobigerina pennyi Bronnimann

2 Rugoglobigerina rugosa (Plummer)

2 Globotruncanella havanensis (Voorwijk)

2 Abathomphalus mayaroensis (Bolli)

Biostratigraphic determination: Globotruncana contusastuartiformis Assemblage Zone, Abathomphalus mayaroensis Subzone. Latest Maastrichtian with reworked species from the G. fornicata-stuartiformis Assemblage Zone (Campanian-early Maastrichtian). 


\section{2-217-22-2 $(80-82 \mathrm{~cm})$}

TA: 5

Gublerina robusta de Klasz

Heterohelix punctulata (Cushman)

Heterohelix striata (Ehrenberg)

Planoglobulina brazoensis Martin

Planoglobulina carseyae (Plummer)

Pseudoguembelina excolata (Cushman)

Pseudoguembelina kempensis Esker

Pseudotextularia deformis (Kikoine)

Pseudotextularia elegans (Rezhak)

Racemiguembelina fructicosa (Egger)

Globotruncana arca (Cushman)

Globotruncana contusa (Cushman)

Globotruncana elevata (Brotzen)

Globotruncana lapparenti Brotzen (Reworked)

Globotruncana linneiana (d'Orbigny) (Reworked)

Globotruncana stuarti (deLapparent)

Globotruncana stuartiformis Dalbiez

Rugoglobigerina pennyi Bronnimann

Rugoglobigerina rugosa (Plummer)

Abathomphalus mayaroensis (Bolli)

Abathomphalus intermedius (Bolli)

Biostratigraphic determination: Globotruncana contusastuartiformis Assemblage Zone, Abathomphalus mayaroensis Subzone. Late Maastrichtian with reworked Campanian-early Maastrichtian.

22-217-22-3 (74-76 cm)

TA: 4

RA

3 Gublerina robusta de Klasz

1 Heterohelix punctulata (Cushman)

1 Heterohelix striata (Ehrenberg)

1 Planoglobulina brazoensis Martin

$1 \quad$ Pseudotextularia deformis (Kikoine)

1 Pseudotextularia elegans (Rzehak)

4 Globotruncana arca (Cushman)

3 Globotruncana elevata Brotzen

2 Globotruncana lapperenti Brotzen (Reworked)

3 Globotruncana linneiana (d'Orbigny) (Reworked)

3 Rugoglobigerina pennyi Bronnimann

1 Abathomphalus mayaroensis (Bolli)

1 Abathomphalus intermedius (Bolli)

Biostratigraphic determination: Globotruncana contusastuartiformis Assemblage Zone, Abathomphalus mayaroensis Subzone. Late Maastrichtian with reworked species from the Campanian-early Maastrichtian.

$$
\text { 22-217-23-1 (78-80 cm) }
$$

TA: 4

RA

4 Gublerina robusta de Klasz

1 Heterohelix sp.

1 Planoglobulina brazoensis Martin

3 Globotruncana arca (Cushman)

2 Globotruncana elevata (Brotzen)

2 Globotruncana lapparenti Brotzen (Reworked)

2 Globotruncana linneiana (d'Orbigny) (Reworked)

3 Rugoglobigerina pennyi Bronnimann

Biostratigraphic determination: Globotruncana contusastuartiformis Assemblage Zone (undifferentiated). Late Maastrichtian.
22-217-23-2 $(80-82 \mathrm{~cm})$

TA: 4

RA

$4 \quad$ Gublerina robusta de Klasz

1 Heterohelix sp.

1 Planoglobulina brazoensis Martin

3 Globotruncana arca (Cushman)

3 Globotruncana elevata (Brotzen)

1 Globotruncana gansseri Bolli

2 Globotruncana lapparenti Brotzen (Reworked)

3 Globotruncana linneaina (d'Orbigny) (Reworked)

3 Rugoglobigerina pennyi Bronnimann

1 Abathomphalus intermedius (Bolli)

Biostratigraphic determination: Globotruncana contusastuartiformis Assemblage Zone, Abathomphalus mayaroensis Subzone. Reworking of Campanian-early Maastrichtian.

22-217-23-3 $(70-72 \mathrm{~cm}) \quad$ TA: 4

RA

3 Gublerina robusta de Klasz

1 Heterohelix striata (Ehrenberg)

1 Heterohelix sp.

$1 \quad$ Planoglobulina sp. aff.P. brazoensis Martin

1 Pseudoguembelina sp.

$1 \quad$ Pseudotextularia elegans (Rzehak)

3 Globotruncana arca (Cushman)

2 Globotruncana contusa (Cushman)

2 Globotruncana elevata (Brotzen)

1 Globotruncana lapparenti Brotzen (Reworked)

2 Globotruncana linneiana (d'Orbigny) (Reworked)

1 Globotruncana stuartiformis Dalbiez

$4 \quad$ Rugoglobigerina pennyi Bronnimann

3 Rugoglobigerina rugosa (Plummer)

2 Globotruncanella havanensis (Voorwijk)

Biostratigraphic determination: Globotruncana contusastuartiformis Assemblage Zone (undifferentiated). Late Maastrichtian with reworking of Campanian-early Maastrichtian.

22-217-23-4 (73-75 cm)

TA: 3

RA

1 Gublerina robusta de Klasz

3 Globotruncana arca (Cushman)

2 Globotruncana lapparenti Brotzen (Reworked)

2 Globotruncana linneiana (d'Orbigny) (Reworked)

$4 \quad$ Rugoglobigerina pennyi Bronnimann

2 Rugoglobigerina rugosa (Plummer)

Biostratigraphic determination: Same as above.

22-217-23-5 (72-74 cm) TA: 4

RA

3 Gublerina robusta de Klasz

4 Globotruncana arca (Cushman)

3 Globotruncana lapparenti Brotzen (Reworked)

3 Globotruncana linneiana (d'Orbigny) (Reworked)

$4 \quad$ Rugoglobigerina pennyi Bronnimann

2 Rugoglobigerina rugosa (Plummer)

2 Abathomphalus mayaroensis (Bolli)

2 Bolivinoides draco (Marsson) 
Biostratigraphic determination: Globotruncana contusastuartiformis Assemblage Zone, Abathomphalus mayaroensis Subzone. Latest Maastrichtian with reworking of late Campanian-early Maastrichtian.

$\begin{array}{cl}\text { 22-217-24-1 (80-82 cm) } & \text { TA: } 5 \\ \text { RA } & \\ 2 & \text { Gublerina robusta de Klasz } \\ 2 & \text { Heterohelix punctulata (Cushman) } \\ 1 & \text { Planoglobulina carseyae (Plummer) } \\ 1 & \text { Ventilabrella multicamerata de Klasz } \\ 2 & \text { Globotruncana arca (Cushman) } \\ 1 & \text { Globotruncana contusa (Cushman) } \\ 2 & \text { Globotruncana lapparenti } \text { Brotzen } \\ 3 & \text { Globotruncana linneiana (d'Orbigny) } \\ 1 & \text { Globotruncana ventricosa White (Reworked) } \\ 4 & \text { Rugoglobigerina pennyi } \text { Bronnimann } \\ 3 & \text { Rugoglobigerina rugosa (Plummer) } \\ 2 & \text { Rugoglobigerina sp. } \\ 3 & \text { Abathomphalus intermedius (Bolli) }\end{array}$

Biostratigraphic determination: Globotruncana contusastuartiformis Assemblage Zone, Abathomphalus mayaroensis Subzone. Late Maastrichtian with reworking of late Campanian-early Maastrichtian.

$$
\text { 22-217-24-2 }(80-82 \mathrm{~cm}) \quad \text { TA: } 4
$$

\section{RA}

4 Gublerina robusta de Klasz

1 Heterohelix punctulata (Cushman)

1 Heterohelix sp.

1 Planoglobulina sp.

1 Pseudoguembelina excolata (Cushman)

4 Globotruncana arca (Cushman)

1 Globotruncana elevata (Brotzen)

1 Globotruncana lapparenti Brotzen (Reworked)

2 Globotruncana linneiana (d'Orbigny) (Reworked)

4 Rugoglobigerina pennyi Bronnimann

2 Rugoglobigerina rugosa (Plummer)

2 Rugoglobigerina sp.

Biostratigraphic determination: Globotruncana fornicatastuartiformis Assemblage Zone, Rugotruncana subcircumnodifer Subzone, R. subpennyi Zonule.

$$
22-217-24-3(80-82 \mathrm{~cm})
$$

TA: 4

\section{RA}

4 Gublerina robusta de Klasz

2 Heterohelix punctulata (Cushman)

$1 \quad$ Heterohelix striata (Ehrenberg)

1 Planoglobulina sp.

4 Globotruncana arca (Cushman)

1 Globotruncana elevata (Brotzen)

1 Globotruncana lapparenti (Blotzen (Reworked)

2 Globotruncana linneiana (d'Orbigny) (Reworked)

1 Globotruncana ventricosa White? (Reworked)

4 Rugoglobigerina pennyi Bronnimann

1 Rugoglobigerina rugosa (Plummer)

1 Rugoglobigerina sp.

1 Globotruncanella havanensis (Voorwijk)

1 Bolivinoides draco (Marsson)

Biostratigraphic determination: Globotruncana fornicatastuartiformis Assemblage Zone, Rugotruncana subcir- cumnodifer Subzone, R. subpennyi Zonule. Early Maastrichtian with reworking of late Campanian.

$22-217-24-4(80-82 \mathrm{~cm})$

TA: 4

\section{RA}

3 Gublerina robusta de Klasz

1 Heterohelix punctulata (Cushman)

1 Heterohelix sp.

1 Planoglobulina sp. ?

4 Globotruncana arca (Cushman)

3 Globotruncana lapparenti Brotzen (Reworked)

2 Globotruncana linneiana (d'Orbigny) (Reworked)

1 Globotruncana ventricosa White (Reworked)

4 Rugoglobigerina pennyi Bronnimann

2 Rugoglobigerina rugosa (Plummer)

1 Rugoglobigerina sp.

Biostratigraphic determination: Same as 217-24-3 (80-82 cm).

\section{2-217-24-5 (75-77 cm)}

TA: 4

\section{RA}

3 Gublerina robusta de Klasz

1 Heterohelix punctulata (Cushman)

1 Heterohelix striata (Ehrenberg)

1 Heterohelix sp.

1 Planoglobulina carseyae (Plummer)

$1 \quad$ Planoglobulina sp.?

1 Pseudoguembelina kempensis Esker

3 Globotruncana arca (Cushman)

3 Globotruncana lapparenti Brotzen

3 Globotruncana linneiana (d'Orbigny)

2 Globotruncana ventricosa White (Reworked)

4 Rugoglobigerina pennyi Bronnimann

2 Rugoglobigerina rugosa (Plummer)

1 Rugoglobigerina sp.

Biostratigraphic determination: Same as 217-24-3 $(80-82 \mathrm{~cm})$.

$$
22-217-24-6(80-82 \mathrm{~cm})
$$

TA: 4

\section{RA}

3 Gublerina robusta de Klasz

1 Heterohelix punctulata (Cushman)

1 Heterohelix striata (Ehrenberg)

$1 \quad$ Heterohelix sp.

1 Planoglobulina carseyae (Plummer)

$1 \quad$ Planoglobulina sp.

3 Globotruncana arca (Cushman)

1 Globotruncana elevata (Brotzen)

1 Globotruncana fornicata Plummer

3 Globotruncana lapparenti Brotzen (Reworked)

3 Globotruncana linneiana (d'Orbigny)

2 Globotruncana ventricosa White (Reworked)

4 Rugoglobigerina pennyi Bronnimann

2 Rugoglobigerina rugosa (Plummer)

1 Rugoglobigerina sp.

Biostratigraphic determination: Same as 217-24-3 (80-82 $\mathrm{cm})$. 
TA: 3

RA

1 Gublerina robusta de Klasz

1 Heterohelix punctulata (Cushman)

1 Heterohelix sp.

$1 \quad$ Pseudotextularia sp.?

1 Globotruncana arca (Cushman)

1 Globotruncana elevata (Brotzen)

1 Globotruncana lapparenti Brotzen

1 Globotruncana linneiana (d'Orbigny)

1 Globotruncana rosetta (Carsey)

1 Globotruncana stuartiformis Dalbiez

1 Globotruncana ventricosa White (Reworked)

3 Rugoglobigerina pennyi Bronnimann

2 Rugoglobigerina rugosa (Plummer)

2 Rugoglobigerina sp.

Biostratigraphic determination: Same as 217-24-3 (80-82 $\mathrm{cm})$.

$$
\text { 22-217-25-3 }(80-83 \mathrm{~cm})
$$

TA: 4

\section{RA}

2 Gublerina robusta de Klasz

1 Heterohelix punctulata (Cushman)

1 Heterohelix striata (Ehrenberg)

1 Planoglobulina sp.?

$1 \quad$ Pseudotextularia sp.

3 Globotruncana arca (Cushman)

3 Globotruncana lapparenti Brotzen

3 Globotruncana linneiana (d'Orbigny)

1 Globotruncana stuartiformis Dalbiez

2 Globotruncana ventricosa White (Reworked)

$5 \quad$ Rugoglobigerina pennyi Bronnimann

$4 \quad$ Rugoglobigerina rugosa (Plummer)

1 Globotruncanella havanensis (Voorwijk)

Biostratigraphic determination: Same as $217-24-3$ (80-82 $\mathrm{cm})$.

$$
\text { 22-217-25-5 }(76-78 \mathrm{~cm}) \quad \text { TA: } 4
$$

RA

1 Gublerina robusta de Klasz

1 Heterohelix punctulata (Cushman)

1 Heterohelix striata (Ehrenberg)

1 Pseudotextularia sp. ?

3 Globotruncana arca (Cushman)

3 Globotruncana lapparenti Brotzen

3 Globotruncana linneiana (d'Orbigny)

2 Globotruncana ventricosa White (Reworked)

$4 \quad$ Rugoglobigerina pennyi Bronnimann

3 Rugoglobigerina rugosa (Plummer)

1 Globotruncanella havanensis (Voorwijk)

Inoceramus prisms abundant

Biostratigraphic determination: Same as 217-24-3 (80-82 $\mathrm{cm})$.

\section{2-217-26-2 (79-81 cm)}

TA: 4

\section{RA}

1 Gublerina robusta de Klasz

1 Heterohelix punctulata (Cushman)

1 Heterohelix striata (Ehrenberg)

1 Pseudotextularia sp. ?
3 Globotruncana arca (Cushman)

3 Globotruncana lapparenti Brotzen

3 Globotruncana linneiana (d'Orbigny)

2 Globotruncana ventricosa White (Reworked)

4 Rugoglobigerina pennyi Bronnimann

3 Rugoglobigerina rugosa (Plummer)

1 Globotruncanella havanensis (Voorwijk)

Inoceramus prisms abundant

Biostratigraphic determination: Same as 217-24-3 $(80-82 \mathrm{~cm})$.

22-217-26-3 (80-82 cm) TA: 4

RA

1 Gublerina robusta de Klasz

1 Heterohelix punctulata (Cushman)

1 Heterohelix striata (Ehrenberg)

1 Pseudotextularia sp.?

3 Globotruncana arca (Cushman)

3 Globotruncana lapparenti Brotzen

3 Globotruncana linneiana (d'Orbigny)

2 Globotruncana ventricosa White (Reworked)

4 Rugoglobigerina pennyi Bronnimann

3 Rugoglobigerina rugosa (Plummer)

1 Globotruncanella havanensis (Voorwijk)

Inoceramus prisms abundant

Biostratigraphic determination: Same as 217-24-3 $(80-82 \mathrm{~cm})$.

$$
\text { 22-217-26-4 (80-82 cm) TA: } 4
$$

RA

1 Heterohelix pulchra (Brotzen)

2 Heterohelix punctulata (Cushman)

1 Heterohelix sp.

$4 \quad$ Globotruncana arca (Cushman)

3 Globotruncana lapparenti Brotzen

3 Globotruncana linneiana (d'Orbigny)

3 Rugoglobigerina pennyi Bronnimann

3 Rugoglobigerina rugosa (Plummer)

Biostratigraphic determination: Same as 217-24-3 $(80-82 \mathrm{~cm})$.

$$
\text { 22-217-26-5 (80-82 cm) TA: } 4
$$

RA

1 Gublerina robusta de Klasz

1 Heterohelix sp.

4 Globotruncana arca (Cushman)

3 Globotruncana lapparenti Brotzen

3 Globotruncana linneiana (d'Orbigny)

$5 \quad$ Rugoglobigerina pennyi Bronnimann

3 Rugoglobigerina rugosa (Plummer)

2 Rugoglobigerina sp.

Biostratigraphic determination: Same zones as 217-24-3 $(80-82 \mathrm{~cm})$.

$$
\text { 22-217-27-2 }(41-43 \mathrm{~cm}) \quad \text { TA: } 2
$$

\section{RA}

1 Heterohelix sp.

$1 \quad$ Guembelitria sp. ?

1 Globotruncana arca (Cushman)

1 Globotruncana lapparenti Brotzen

1 Globotruncana linneiana (d'Orbigny) 
1 Rugoglobigerina rugosa (Plummer)

1 Globotruncanella sp.

Flood of inoceramus prisms

Biostratigraphic determination: Same as 217-24-3 $(80-82 \mathrm{~cm})$.

$22-217-27-3(71-73 \mathrm{~cm})$

TA: 4

RA

2 Gublerina robusta de Klasz

$1 \quad$ Heterohelix pulchra (Brotzen)

2 Heterohelix punctulata (Cushman)

$1 \quad$ Heterohelix sp.

$1 \quad$ Heterohelix striata (Ehrenberg)

$1 \quad$ Planoglobulina sp.

1 Pseudoguembelina sp.

1 Globotruncannella mattsoni (Pessagno)

2 Globotruncana arca (Cushman)

3 Globotruncana lapparenti Brotzen

3 Globotruncana linneiana (d'Orbigny)

4 Rugoglobigerina pennyi Bronnimann

2 Rugoglobigerina rugosa (Plummer)

2 Rugoglobigerina sp. aff. R. milamensis Smith and Pessagno

1 Globotruncanella sp.

Biostratigraphic determination: Early Maastrichtian. Same as $217-24-3(80-82 \mathrm{~cm})$.

\section{2-217-28-1 (66-68 cm) TA: 2}

RA

1 Globotruncana lapparenti Brotzen?
$1 \quad$ Rugoglobigerina sp.
Inoceramus prisms are common. Preservation very bad. Biostratigraphic determination: Early Maastrichtian via superposition.

22-217-28-2 $(86-88 \mathrm{~cm}) \quad$ TA: 2

RA

2 Rugoglobigerina sp.

1 Rugoglobigerina pennyi Bronnimann

1 Gublerina robusta de Klasz

Inoceramus prisms are common. Preservation very bad.

Biostratigraphic determination: Early Maastrichtian via superposition.

22-217-30-1 (106-108 cm) TA: 0

Barren

$22-217-30-2(85-87 \mathrm{~cm}) \quad$ TA: 0

Barren

\section{SUMMARY-SITE 217}

1. The interval from Core 17 , Section $1,68-70 \mathrm{~cm}$, to Core 24 , Section $1,80-82 \mathrm{~cm}$, is assignable to the latest Maastrichtian, Abathomphalus mayaroensis Subzone.

2. The interval from Core 24 , Section $2,80-82 \mathrm{~cm}$, is assignable to the early Maastrichtian, Rugotruncana subcircumnodifer Subzone, Rugotruncana subpennyi Zonule.

3. If the Globotruncana gansseri Subzone is present at Site 217, it is present between Core 24, Section 1, 80-82 $\mathrm{cm}$, and Core 24 , Section $2,80-82 \mathrm{~cm}$.

4. Reworking of late Campanian-early Maastrichtian elements is common in the latest Maastrichtian. 